\title{
Review of: "Development of diabetes mellitus following hormone therapy in prostate cancer patients is associated with early progression to castration resistance"
}

\author{
Shingo Hatakeyama ${ }^{1}$
}

1 Hirosaki University

Potential competing interests: The author(s) declared that no potential competing interests exist.

The authors evaluated the impact of post treatment DM development and PSA on the progression to CRPC in patients undergoing hormone therapy and concluded that development of diabetes mellitus following hormone therapy in prostate cancer patients is associated with early progression to castration resistance. However, the reviewer could not agree with this conclusion based on the study design and outcomes.

The critical points are follows:

1 ) the number of patients with postDM $(n=8,3.5 \%)$ were to small to find clinical significance. It is very hard to believe the finding of this study. The authors need to learn the feasible numbers of events for statistical analysis.

2) The finding of "PSA $\geq 18$ were significantly associated with the progression to CRPC" are not useful in this mixed cohort. The impact of $P S A \geq 18$ should be completely different in patients with or without metastasis. As this cohort included 187 (81.3\%) of M0 disease, the authors need to focus on M0 disease alone. From the results of this study, all patients had higher risk for CRPC progression because patients with M1 disease should have higher PSA( $>18)$. This finding makes no sense.

In conclusion, the authors need to include urologist for analysis to find useful outcomes. The quality of the present study was too terrible, and not suitable for publication. 